\title{
Ground water level monitoring device on marshland
}

\author{
Jacek Rapinski $^{\mathrm{a}}$, Kamil Kowalczyk ${ }^{\mathrm{a}}$, Michal Smieja ${ }^{\mathrm{b}}$ \\ ${ }^{a, b}$ Department of Geodesy, University of Warmia and Mazury in Olsztyn, Oczapowskiego 1, 10-719 Olsztyn, Poland \\ ${ }^{b}$ Chair of Mechatronics, University of Warmia and Mazury in Olsztyn, Sloneczna 46a, 10-719 Olsztyn, Poland
}

\begin{abstract}
This paper presents the overall concept of a design of a multi-sensor water level measurement device. Requirements, possible applications and design schemes are presented and described. Some basic considerations on the possible equipment, electronic parts and algorithms are also included.
\end{abstract}

Keywords: piezometer; ground water level.

\section{Introduction}

Changes in groundwater level are caused by factors arising from activity the forces of nature and of the expansion of human activity (so-called anthropogenic changes). Changes resulting from the first factor are long-lasting and virtually unnoticeable in short periods of observation. In contrast, anthropogenic changes can occur rapidly, and thus have a shortterm nature. Permanent monitoring of groundwater level makes it possible to assess the scale of this phenomenon, as well as the selection of the method of counteracting of soil drying or hydration. Changes in groundwater level have a direct impact on agricultural and forestry activities, as well as construction, spatial planning, nature conservation, and many other areas of life.

In Europe, many researchers from the field of geodesy, hydrology, agriculture and biology address this issue. Widely described issues of the impact of changes in groundwater can be found for example in [4].

Monitoring of groundwater can be carried out in many ways. For this purpose, among other things satellite measurements can be used. Data from these measurements are collected and processed in a specially created research centers of meteorology and geology such as European Centre for Medium-Range Weather Forecasts, Helmholt-Zentrum Potsdam Deutsches GeoForschungsZentrum or Global Land Data Assimilation System [5].

These data are rather global than regional. As a regional solution systems like Satellite Water Quality Monitoring based on satellite imagery are used. Locally, on open areas, monitoring of groundwater level changes can be carried out using, eg, radar interferometry. In places where it is impossible to apply this methods or it is uneconomic, measurements are made cyclically directly in the field. For this measurements piezometers are used. Usually they are conducting the surveys on the basis of the measurement of the excess of pore water pressure, or they are built as a set of few sensors collecting informations about groundwater level, pressure, conductivity and temperature.

The scope of such measurements depends on the needs of the researcher and the equipment available and sometimes also on the availability of land (thaw, wetlands, bogs, etc.). The best solution would be to install a set of permanent devices at the measurement points and to automate the measuring process. This is the pronciple on which sensors made by Stevens or Hydromet are built. The collected data are transmitted via telecommunication solutions to the recipient.

Monitoring of water level is an important topic in many aspects of hydrology, environmental engineering and even in public safety. Data from both surface and ground water levels can be used in science and in normal life. For example ground water level monitoring can be used in geodesy to compensate gravimetric surveys for ground water level influence. There is a lot of various water level sensors available on the market. They differ in accuracy, range, cost and possible application. The main disadvantage of most of them is the lack of automatic compensation for vertical movement of sensor's anchor, which can be caused by many different reasons (eg. ground freezing, building sedimentation). In this paper the automatic system for water level monitoring with sensor's anchor vertical movement compensation is proposed. These arrangements

Corresponding author: Michal Smieja. E-mail address: smieja@uwm.edu.pl

http://dx.doi.org/10.3846/enviro.2014.089

(C) 2014 The Authors. Published by VGTU Press. This is an open-access article distributed under the terms of the Creative Commons Attribution License, which permits unrestricted use, distribution, and reproduction in any medium, provided the original author and source are credited. 
do not take into account the vertical displacement of sensors (or sensor anchors) themselves which in effect may disturb the interpretation of results. A better solution would be to introduce an additional element to allow the monitoring of movements of these devices.

\section{Ground water level measurement techniques on marshland}

The range of ground water level surveys is strongly dependent on good hydro geological recognition of catchment. To measure the free water level one can use points where the water level is accessible like drill holes, wells, ditches, shafts etc. or special observation wells - piezometers (Fig. 1). Open drilled piezometer enables, beside water level, measurement of physical and chemical parameters of water. It also allow to pick up water samples for chemical analysis in laboratory. Piezometer depth depends on local geological conditions, since it's filter must be inside the water-bearing layer.

In case of marshlands, especially with quaking bog, mounting of a piezometer can be difficult. Bottom part of a piezometer must be placed in mineral soil, which should prevent the piezometer from raising subsiding with water level, which would bring outliers into measurements. Top part of piezometer must be secured with protective well and sealed 1 . To properly measure water level in reference to national height system, a stable benchmark is required in the vicinity of piezometer.

To measure water level one can use:

- leveling rod;

- float;

- sounder;

- lighter;

- water gauge (analogue or digital).

The most common and simplest water level measurement device is a sounder hang on a measuring tape (with respect to the size of a device). The accuracy of such measurement is usually $1 \mathrm{~cm}$ in reference to a marker on piezometer, maximum depth is usually $50 \mathrm{~m}$. The marker for sounder measurements must be stable and its height must be determined. The measurement is made by lowering the measurement device on the tape into the well. When the sound is heard the tape must be picked up by few centimeters and lowered again few times to precisely determine a water level.

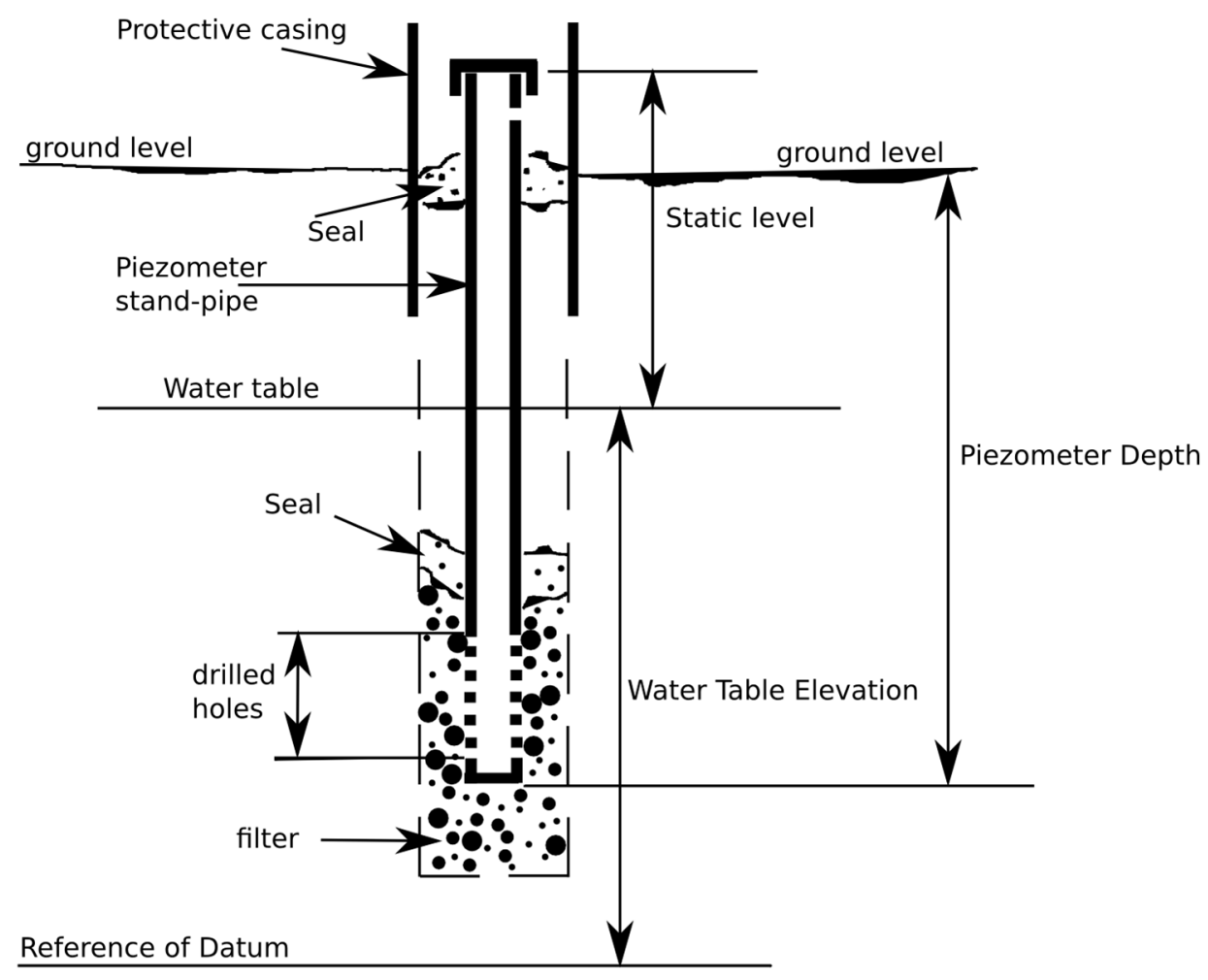

Fig. 1. Outline of piezometer

When the depth of a well is grater then $50 \mathrm{~m}$, a lighter can be used. The principle of measurement is similar to sounder. Main difference is that instead of sound, a light is lit when the device is merged into the water. Using this two techniques the results of measurement must be manually written by user. Ground water level fluctuates with seasons. It is caused by variable water inflow and outflow volume, either natural or forced. The size of these changes and it's seasonal dynamics make a basis for ground water distribution and determination of characteristic states which are: monthly and half-year and year mean and extreme states. The water level amplitude is a common measure that characterize ground water level dynamics. 


\section{Overall idea of ground water level measurement device}

To obtain a reliable water level in a certain height system the height of the measurement device fixed point must be known. This can be done by mounting the device to the firm sediment e.g. bedrock. In the case when there is no possibility to reach a bedrock or it is too expensive, the fixed point height must be determined continuously. To complete this task one can use either automated laser level (if there is a reference point nearby) or a GNSS positioning. The fastest way would be to use a GPS RTK grade receiver, but since geodetic, hi-precision GPS devices are very expensive, there is a need to use a cheaper device for monitoring of the ground water level height.

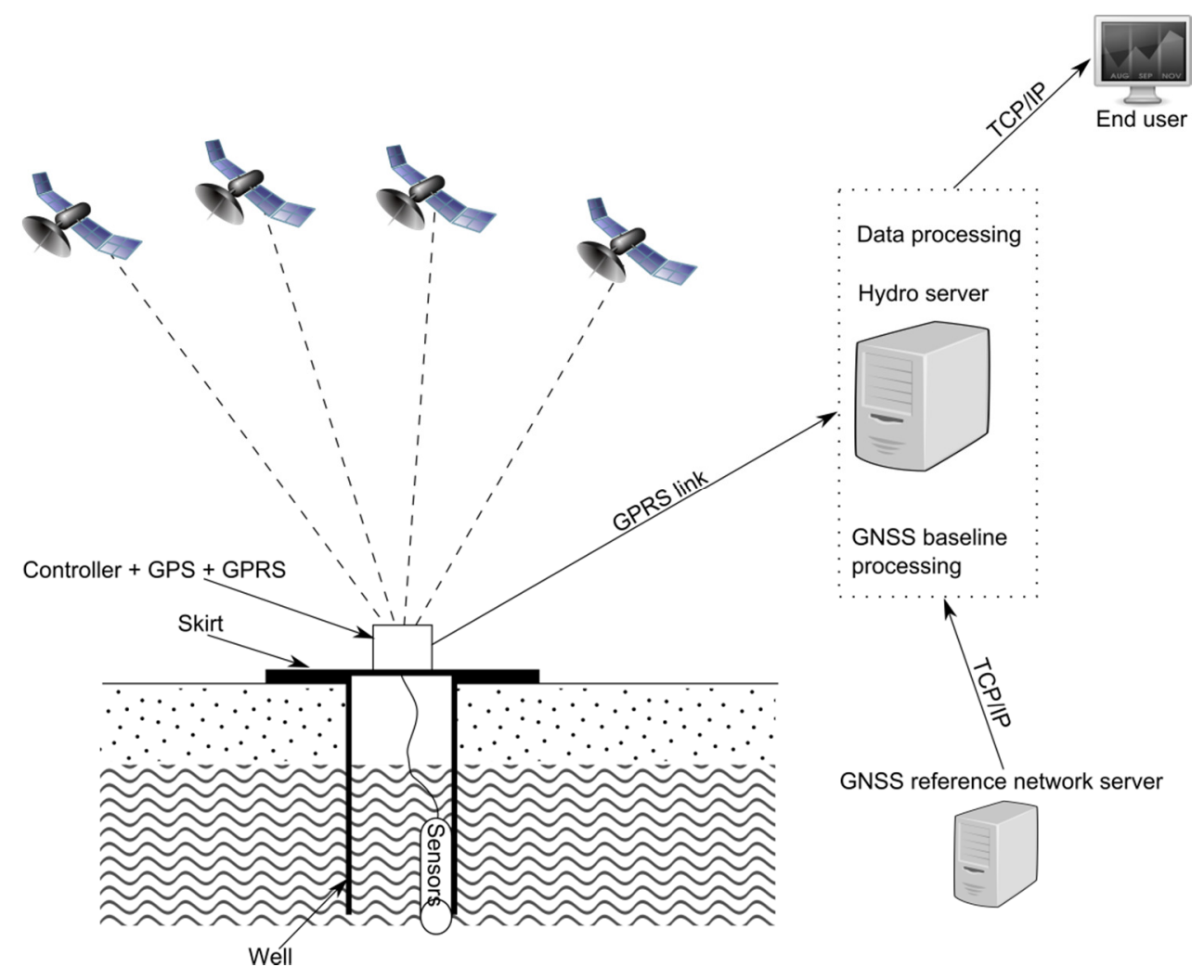

Fig. 2. The idea of the system

The most precise GNSS technique is a static survey [3]. It is based on the large amount of collected data post processed in a reference to another (fixed) points. To obtain relatively good position the carrier phase observations must be used. Therefore the use of navigation GPS receivers (e.g. SirfStar chipset based devices) is not possible. Hence there is a choice of small, cheap GNSS chipsets with the possibility to log L1 phase data available on market. For example uBLOX LEA 6T. Since changes in the devices height is not rapid, there is a possibility to collect data for post processing. Figure 2 depicts the overall idea of the measurement system.

\section{Level design and integration with GPS}

\subsection{Determination of reference height for the water level measurements}

Integration with a GNSS receiver allows to obtain a very useful information about device's position and height. The height data is of a particular impor $\neg$ tance, thus it can be treated as a reference point for the water level measurement. There is a lot of possibilities when chosing an optimal GPS positioning strategy. Most important features that needs to be taken into consideration are:

- accuracy of positioning;

- required hardware (e.g. single or double frequency, GPS, GLONASS);

- wireless communication availability;

- energy consumption;

The device equipped with GNSS receiver and communication device such as a GPRS/UMTS modem can calculate its position in few modes [1]:

- autonomous;

- static;

- DGPS (Differential GPS);

- RTK (Real Time Kinematics). 
First method is a single - receiver, lowest accuracy method used mostly in navigation. Since the positioning accuracy is of tens of meter, it is not suitable for this kind of tasks. The DGPS method is a differential method that uses two receivers (rover and base) but it's accuracy is of tens of centimeters to 2 meters which is also too low. Two methods that needs to be considered are static and RTK. RTK is a most frequently used method in modern surveying. It provides a position with the accuracy of 3 to $5 \mathrm{~cm}$ almost in real time. The accuracy in horizontal plane is usually about 3 time better then height accuracy. This method requires a communication link with another GPS receiver placed on the point with known coordinates. Nowadays it is common to use CORS (Continously Operating Reference Stations), so there is no need to own a reference station, still communication must be provided. On marshland the only reasonable way to provide a communication is GSM/GPRS. The major advantage of this method is a possibility to obtain immediate position. The accuracy of this method is close to the required limits.

Static method is based on collecting satellite observables through time with selected interval (e.g. one observation every minute). By extend of the length of the measurement session, the increase in the accuracy can be obtained [1]. Since in the static surveys the position calculation is done in post processing various algorithms and error models can be used to obtain best possible accuracy. This approach is the most accurate of all, it allows to calculate position with accuracy below $1 \mathrm{~cm}$ in both vertical and horizontal planes [2].

Since the changes in the reference point height are not rapid, static method is favorable in this application as it is the most accurate and does not require constant communication with reference station.

\subsection{Design of the measuring device}

A draft of the devices design is presented in Figure 3. It depicts a way of required tasks realization:

1. gathering and pre-processing of data;

2. managing GPS and GPRS interfaces;

3. power managements;

4. auto-diagnostics and information about state of the device.

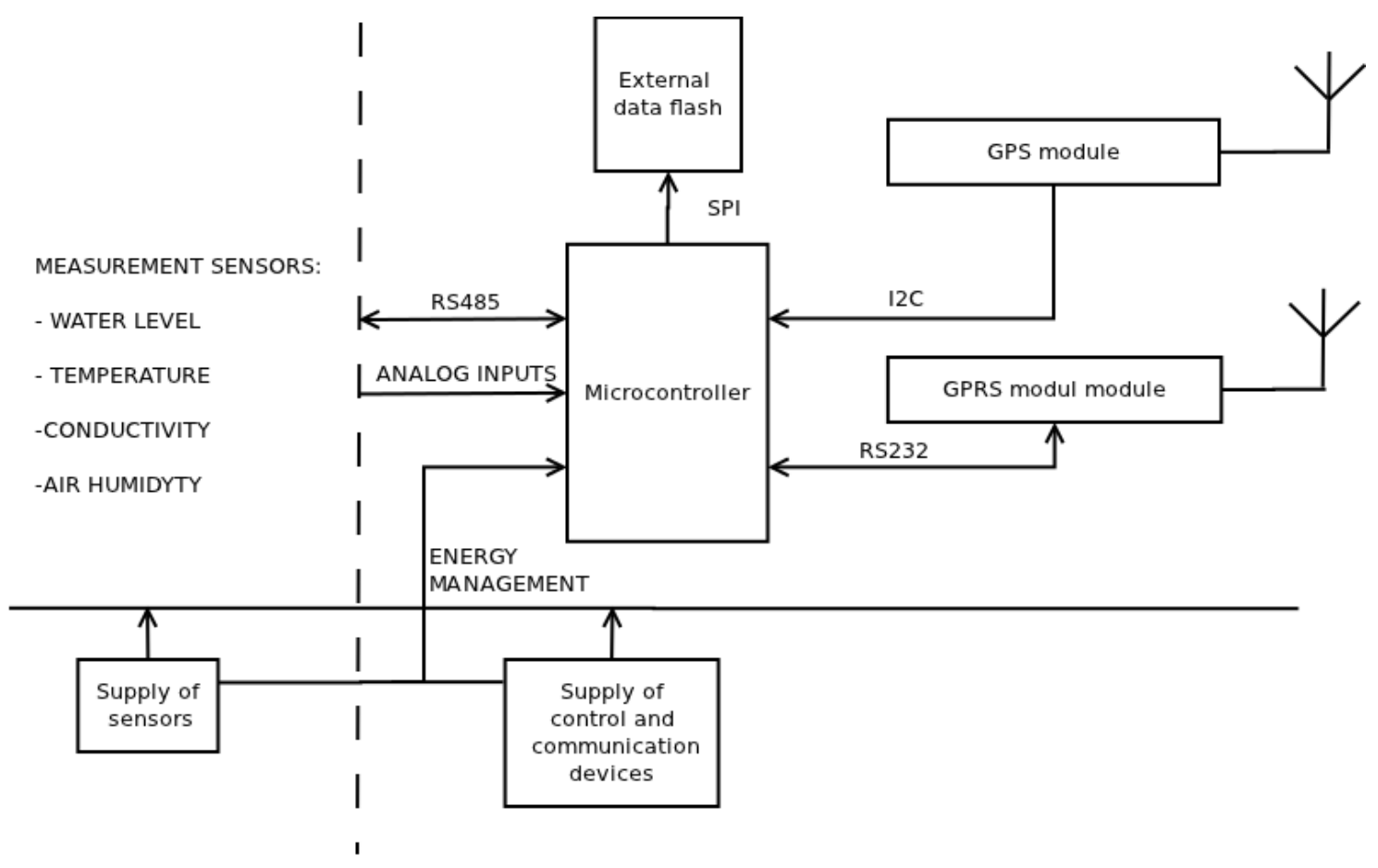

Fig. 3. The outline of system's electronics

The device is designed for long term operation in harsh environment. In many cases, considering the device location on marshlands, the access to it is difficult. Therefore the design ensures a buffering and storage of data on local flash memory parallel to the GPRS transmission.

One of the major problems in case of electronic devices is to provide battery supply in low temperatures. Therefore the sensors and controllers will be powered from separate cells. In addition to prevent negative temperatures, energy source will be placed under ground (or underwater) below freezing level in waterproof casing.

\subsection{Possible sensors}

Following values are available for measurements:

- water level sensor; 
- temperature at the sensor;

- temperature above the water;

- water electrical conductivity;

- air humidity.

Water level sensor is a differential pressure sensor that can measure water level in a reference to a fixed point. The use of a pressure sensor will prevent the sensors failure in winter, when floating sensors would freeze. This sensor is equipped with electronic thermometer, so the temperature at the depth of a sensor is available. Any number of additional temperature sensors can be added to the device, both under and over the water level.

\section{Conclusions}

The device is currently in the development stage. Sensors applied in the device allows to gather information on the temperature of underground water, the pressure of the air and water, conductivity and temperature. GPS receiver and server via the GSM / GPRS network allows for constant monitoring of measured parameters and movements of mounted devices. Adding rangefinder reflector and leveling rod to the device allows for alternative to GPS measurement in the field. This is particularly important when measuring position (sensors) is located in the area without open horizon or in wetlands, where direct access to the device is difficult for a large part of the year. Use of these devices allows observation of movements of of sensors from a convenient position for the observer. Additional GSM / GPRS transmitter allows to quickly receive information on the current parameters and model its change. In 2014 it is planned to install three of these devices at the test area along with the development of the system of collecting, cataloging and visualization of the collected information The proposed use of geodetic techniques for high precision measurement and increasing the number of of sensors in relation to the standard solutions gives a significant advantage of this concept over the commonly used solutions..

The possibility of placing cheap, multipurpose sensors in selected places of the marchland will allow to perform multiple spatial analysis of a water level, temperature distribution along with its dynamics. Connection with a server through GSM/GPRS will allow automatic collection of data as well as obtaining data "on demand".

\section{References}

[1] Del Re, E.; Ruggieri, M. (Eds.). Satellite Communication and Navigation Systems. Springer Science, 2008.

[2] Hoffmann-Wellenhof, B. Global Positioning System Theory and Practice, Springer-Verlag, 1992. http://dx.doi.org/10.1007/978-3-7091-5126-6

[3] Leick, A. GPS' Satellite Surveying. John Wiley \& Sons, Inc., 1995.

[4] Batelaan, O.; De Smedt, F.; Triest, L. 2003. Regional groundwater discharge: phreatophyte mapping, groundwater modelling and impact analysis of land-use change, Journal of Hydrology 275(1-2): 86-108. http://dx. doi.org/10.1016/S0022-1694(03)00018-0

[5] Werth, S. 2010. Calibration of the global hydrological model WGHM with water mass variations from GRACE gravity data. Faculty of Matematics and Natural Sciences at the University of Postam, Postam. 